\title{
Th17 cytokines: novel potential therapeutic targets for COPD pathogenesis and exacerbations
}

\author{
Olivier Le Rouzic (1) 1 2,3,4,5 , Muriel Pichavant ${ }^{1,2,3,4}$, Emilie Frealle 1 2,3,4,6 \\ Antoine Guillon $7,8,9$, Mustapha Si-Tahar $\mathbb{1}^{8,9}$ and Philippe Gosset (1) 1,2,3,4
}

Affiliations: 'Université de Lille, U1019 - UMR 8204, Lung Infection and Innate Immunity, Center for Infection and Immunity of Lille (CIIL), Lille, France. ${ }^{2}$ CNRS, UMR 8204, Lille, France. ${ }^{3}$ INSERM, U1019, Lille, France. ${ }^{4}$ Institut Pasteur de Lille, Lille, France. ${ }^{5}$ Service de Pneumologie Immunologie et Allergologie, CHU Lille, Lille, France. ${ }^{6}$ Laboratoire de Parasitologie et Mycologie Médicale, CHU Lille, Lille, France. ${ }^{7}$ Service de Réanimation Polyvalente, CHRU de Tours, Tours, France. ${ }^{8}$ Inserm, U1100 - Centre d'Etude des Pathologies Respiratoires, Tours, France. ${ }^{9}$ Université François Rabelais, Tours, France.

Correspondence: Philippe Gosset, INSERM U1019-CNRS UMR8204, CIIL, Lung Infection and Innate Immunity Team, Institut Pasteur de Lille, 1 Rue du Professeur Calmette, BP245, F-59019 Lille cedex, France.

E-mail: philippe.gosset@pasteur-lille.fr

@ERSpublications

Alteration of IL-17 and IL-22 production plays a key role in COPD physiopathology and development of exacerbations http://ow.ly/zKFj30elgdw

Cite this article as: Le Rouzic O, Pichavant M, Frealle E, et al. Th17 cytokines: novel potential therapeutic targets for COPD pathogenesis and exacerbations. Eur Respir J 2017; 50: 1602434 [https://doi.org/10.1183/ 13993003.02434-2016].

ABSTRACT Chronic obstructive pulmonary disease (COPD) is a chronic inflammatory disease of the airways caused mainly by cigarette smoke exposure. COPD progression is marked by exacerbations of the disease, often associated with infections. Recent data show the involvement in COPD pathophysiology of interleukin (IL)-17 and IL-22, two cytokines that are important in the control of lung inflammation and infection. During the initiation and progression of the disease, increased IL-17 secretion causes neutrophil recruitment, leading to chronic inflammation, airways obstruction and emphysema. In the established phase of COPD, a defective IL-22 response facilitates pathogen-associated infections and disease exacerbations. Altered production of these cytokines involves a complex network of immune cells and dysfunction of antigen-presenting cells. In this review, we describe current knowledge on the involvement of IL-17 and IL-22 in COPD pathophysiology at steady state and during exacerbations, and discuss implications for COPD management and future therapeutic approaches.

Received: Dec 122016 | Accepted after revision: July 142017

Conflict of interest: Disclosures can be found alongside this article at erj.ersjournals.com 


\section{Introduction}

Chronic obstructive pulmonary disease (COPD) is a chronic inflammatory disease of the airways caused mainly by exposure to noxious particles or gases and characterised by persistent airflow obstruction [1] COPD remains a major cause of morbidity and mortality worldwide. The number of COPD cases is still increasing and was estimated to be 384 million in 2010, with a global prevalence of $11.7 \%$ [2]. It will be the third greatest cause of death worldwide by 2030, according to the World Health Organization. Cigarette smoking is the main risk factor for the development of COPD, although other factors, including indoor and outdoor pollution and genetic determinants, are known to be involved. The disease is characterised by persistent airway limitation that is usually progressive and punctuated by clinical exacerbations mainly related to bacterial and viral infections [3]. These exacerbations have a strong impact on health status, exercise capacity, lung function and mortality $[4,5]$. Clinical symptoms of the disease are mostly related to lung chronic inflammation, an alteration of airway structure leading to airflow limitation and the destruction of the alveolar wall, leading to emphysema. Understanding the underlying immune mechanisms responsible for disease progression as well as the associated susceptibility to respiratory infections is crucial to guide future therapeutics. As T-helper cell (Th) 17 cytokines play a key role in driving mucosal immune responses, this review focuses on the role of Th17 cytokines, interleukin (IL)-17 and IL-22 in the pathophysiology of COPD, namely chronic inflammation, airway obstruction and emphysema (figure 1). In addition, we review their role during exacerbations of COPD, particularly in susceptibility to infection [6].

\section{Search strategy and selection criteria}

We searched PubMed for articles published between January 1990 and May 2017, using the term "COPD" combined with the following individual search terms: "Th17", "IL-17" or "IL-22". Articles in English and French from these searches and relevant references cited therein were reviewed.

\section{Th17 immunity in the lung}

Th17 cells represent a recently described T-cell subset, differentiated from naïve $\mathrm{CD} 4^{+} \mathrm{T}$-cells stimulated with antigen-presenting cells (APCs) primed with antigen and in the presence of IL- 6 and IL-1 $\beta$ in humans or IL-6 and transforming growth factor (TGF)- $\beta$ in mice [7]. IL-23 secondarily promotes complete differentiation and sustains IL-17A production. Not only Th17 cells, but also innate cells, such as $\gamma \delta \mathrm{T}$-cells, type 3 innate lymphoid cells (ILC3), natural killer (NK) cells or natural killer T-cells (NKT) produce IL-17A, IL-17F and IL-22, which are key cytokines in the host immune response against mucosal infections $[6,8]$. The IL-17 family consists of six members, but antimicrobial activity is associated mainly with IL-17A, IL-17F and IL-17A/F heterodimer members [9]. The receptor for IL-17A, IL-17F and IL-17A/F is the IL-17 receptor (IL-17R)A-IL-17RC complex. Like IL-19 and IL-20, IL-22 is a member of the IL-10 family, and can be coexpressed with IL-17A [10]. The IL-22 receptor is a heterodimer receptor complex composed of IL-22 receptor subunit 1 (IL-22RA) and IL-10R2 [11]. Activation of IL-17 or IL-22 receptors expressed on bronchial epithelial cells induces secretion of neutrophil chemotactic factors such as granulocyte colony-stimulating factor or the chemokine CXC ligand (CXCL)8, cytokines such as IL-6 and antimicrobial peptides such as $\beta$-defensins and S100 proteins $[7,10]$. Moreover, exposure of human bronchial epithelial cells to IL-17A stimulates bicarbonate and chloride secretion, resulting in the alkalinisation of liquid on the mucosal surface of polarised cells [12]. Thus, IL-17A can facilitate the antibacterial activity of the mucus fluid, since its acidification in inflamed lungs alters its antibacterial activity [13]. IL-22 contributes to tissue protection and regeneration by inducing mucus-associated protein secretion from goblet cells and the proliferation of epithelial cells [10]. However, neutrophil recruitment is mainly related to IL-17A and IL-17A/F, as it is not affected by neutralisation of IL-17F or IL-22, suggesting a specific role of each cytokine, which does not exclude cytokine interactions [14]. In particular, during bacterial infection, IL-22 synergises with IL-17A to induce antimicrobial peptides and chemokine production [15].

Remarkably, these two cytokines have both beneficial and deleterious effects that depend on their relative production [16]. Therefore, differential expression of IL-17A and IL-22 is tightly regulated by different transcriptional factors including STAT3, retinoid-related orphan receptor (ROR) $\gamma \mathrm{t}$, c-Maf and aryl hydrocarbon receptor (AHR), which are activated by different combinations of cytokines including IL-1 IL-6, IL-23 and TGF- $\beta$ [10]. The development of Th17 cells comprises three overlapping steps: differentiation, amplification and stabilisation. The differentiation is dependent on IL- 6 and TGF- $\beta$. IL-21 produced by developing Th17 cells induces cell replication and IL-23 stabilises these differentiated Th17 cells. Two types of Th17 cells induced by TGF- $\beta 1+$ IL- 6 or IL- $1 \beta+$ IL- 6 + IL- 23 can be generated from naïve T-cells, and are functionally and transcriptionally distinct [17]. Moreover, IL-10 is able to regulate Th17 cell immunopathology and to reduce autoimmunity, whereas restimulation in the presence of IL-23 confers pathogenic functions to Th17 cells [18]. This suggests an important driving role of APCs which 


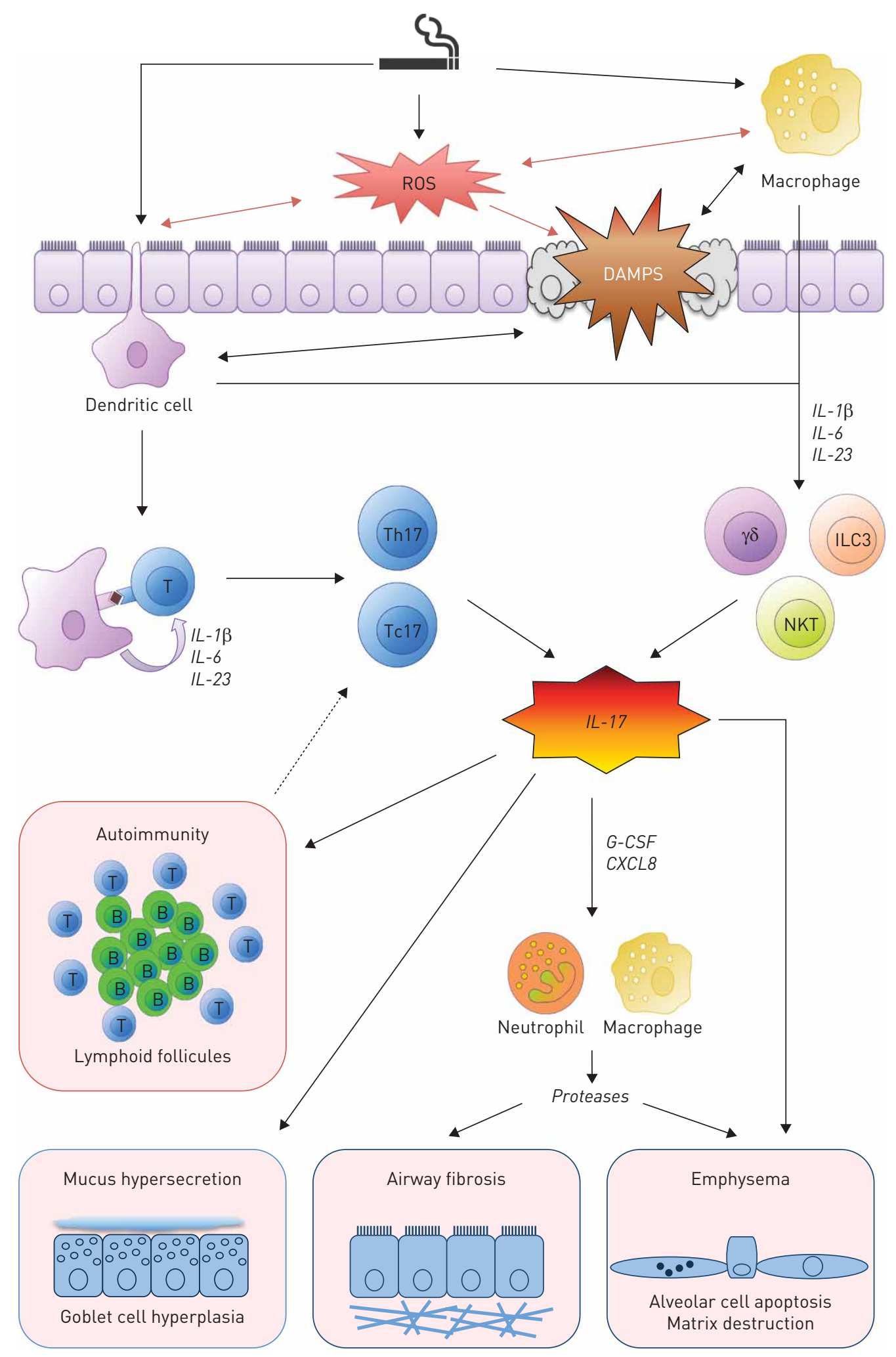

FIGURE 1 Interleukin (IL)-17 in the pathophysiology of stable chronic obstructive pulmonary disease. ROS: reactive oxygen species; DAMPS: damage-associated molecular patterns; T: T-cell; B: B-cell; $\gamma \delta$ : $\gamma \delta$ T-cell; ILC3: innate lymphoid cell type 3; NKT: natural killer T-cell, G-CSF: granulocyte colony-stimulating factor; CXCL8: interleukin 8.

contributes to the secretion of these pro-Th17 cytokines [10]. While recent data suggest that protective and pathogenic Th17 cells may represent specific T-cell phenotypes, there are no data to support the idea that only phenotypically pathogenic Th17 cells are increased in COPD [19]. 


\section{IL-17 and IL-22 cytokines in COPD chronic inflammation}

In most studies, blood IL-17A concentrations in COPD patients are higher compared with healthy nonsmokers and with healthy smokers [20-25] (table 1). In contrast, blood IL-22 concentration is elevated in both COPD and healthy smokers compared to healthy nonsmokers [23, 24]. The blood concentration of IL-17A, but not IL-22 increases with COPD severity and is negatively correlated with airflow limitation measured by forced expiratory volume in $1 \mathrm{~s}$ (FEV1). This is associated with higher proportions of Th17 cells and IL-17-producing $\mathrm{CD} 8^{+} \mathrm{T}$-cells $(\mathrm{Tc} 17)$ in the blood of COPD patients compared to healthy nonsmokers and healthy smokers; these numbers are related to airflow limitation severity [22, 23, 26-34]. Interestingly, Th1 cells are elevated in blood from COPD and healthy smokers, but are not correlated with airway obstruction, suggesting that systemic Th17 inflammation may be more relevant in COPD pathophysiology $[32,33]$.

Within the airways, quantification of Th17 cytokines in the sputum of COPD patients showed higher IL-17A concentrations that correlated with the concentration of neutrophil chemotactic mediators CXCL8 and IL-6, as well as with airway obstruction $[24,27,28,35]$ (table 2). However, the number of other studies with conflicting data or Th17 cytokine levels below the limit of detection demonstrates the low reproducibility of results based on sputum analysis and/or that the distribution of Th17 cytokines is restricted to the tissue [3640]. Indeed, biopsy-based studies have demonstrated the association of the disease with higher numbers of Th17 cytokine-positive cells in the airway walls and the lungs [26, 37, 38, 41-46] (table 3). Immunostaining of bronchial biopsies showed higher IL-17A-, IL-17F- and IL-22-positive cells in COPD patients, including endothelial cells [43]. As suspected, Th17 and Tc17 cells are more frequent throughout bronchial airways and in lung alveolar walls of COPD patients $[41,46]$. But in small airways, IL-17A- and IL-17F-positive cells include not only T-cells but also neutrophils, mast cells and B-cells, demonstrating the multiple potential cell sources of these cytokines in the disease [38]. Therefore, the key step in COPD pathophysiology, and particularly neutrophilic inflammation, seems to be strongly dependent on IL-17A.

The formation of bronchial-associated lymphoid tissue is a characteristic feature of COPD pathophysiology [47]. IL-17A contributes to the development of lymphoid follicles via activation of CXCL12 and CXCL13 production [48, 49], but IL-17A-positive cells are found in their periphery [38]. In contrast,

TABLE 1 Main results of studies focusing on interleukin (IL)-17 and IL-22 concentrations and T-helper type 17 cell and IL-17-producing CD8 ${ }^{+}$T-cell proportions in the blood of chronic obstructive pulmonary disease (COPD) patients

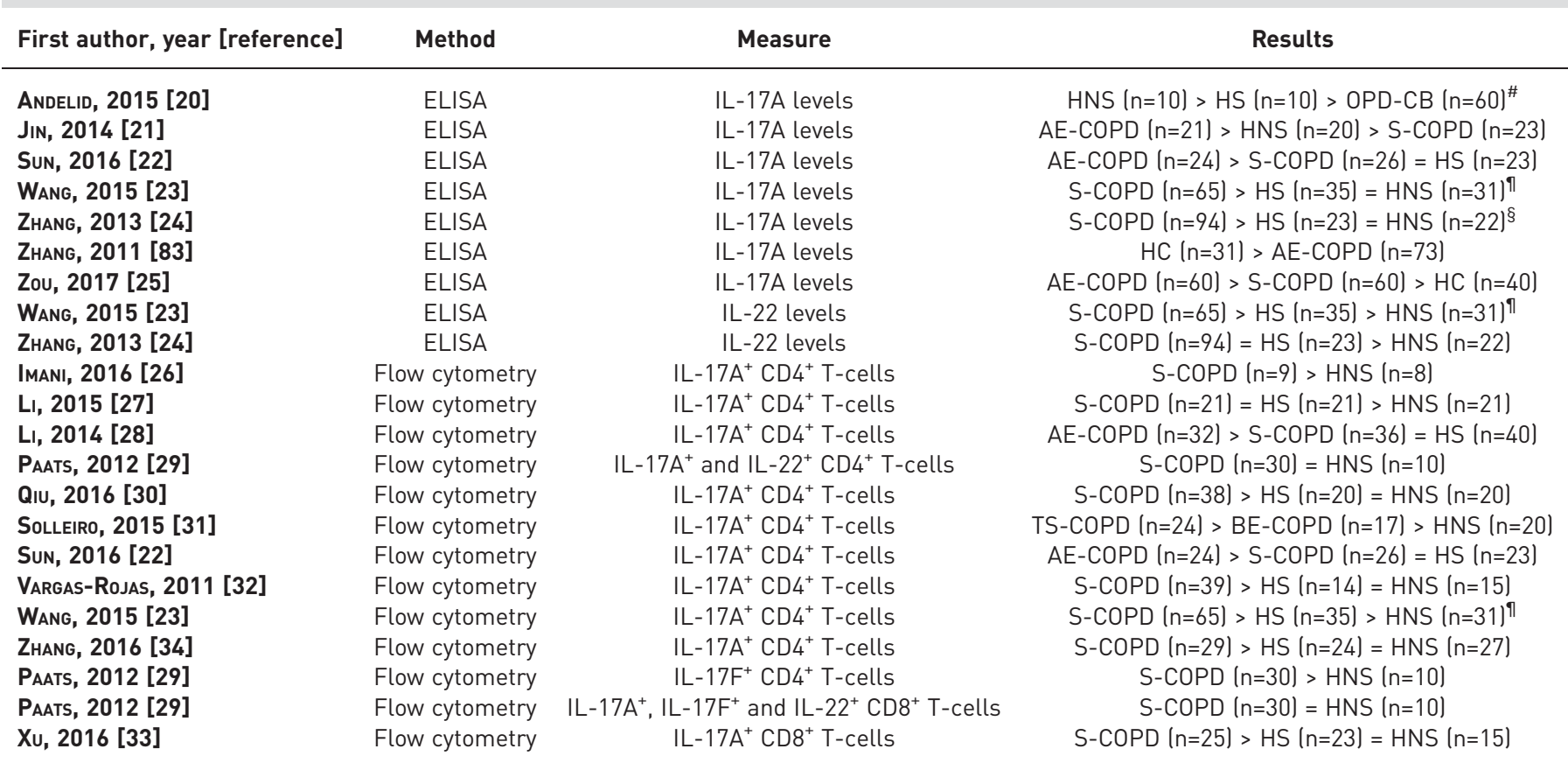

Data are presented as comparisons of cytokines levels or cells proportions in the blood of healthy nonsmokers (HNS), healthy smokers (HS) and patients with COPD. OPD-CB: obstructive pulmonary disease including chronic bronchitis; AE-COPD: acute exacerbation of COPD; S-COPD: stable COPD; HC: healthy controls without data on smoking status; TS-COPD: COPD related to tobacco smoking; BE-COPD: COPD related to biomass exposure. ${ }^{\#}$ : HNS is significantly different from OPD-CB, but HS is not significantly different from the two other groups; ${ }^{\text {? }}$, higher cytokine levels or cell proportions in severe/very severe COPD compared to mild/moderate COPD; ${ }^{\S}$ : IL-17A levels increase with COPD stages. 
TABLE 2 Main results of studies focusing on interleukin (IL)-17 and IL-22 concentrations and T-helper cell type 17 and IL-17-producing CD8 ${ }^{+}$T-cell proportions in the sputum of chronic obstructive pulmonary disease (COPD) patients

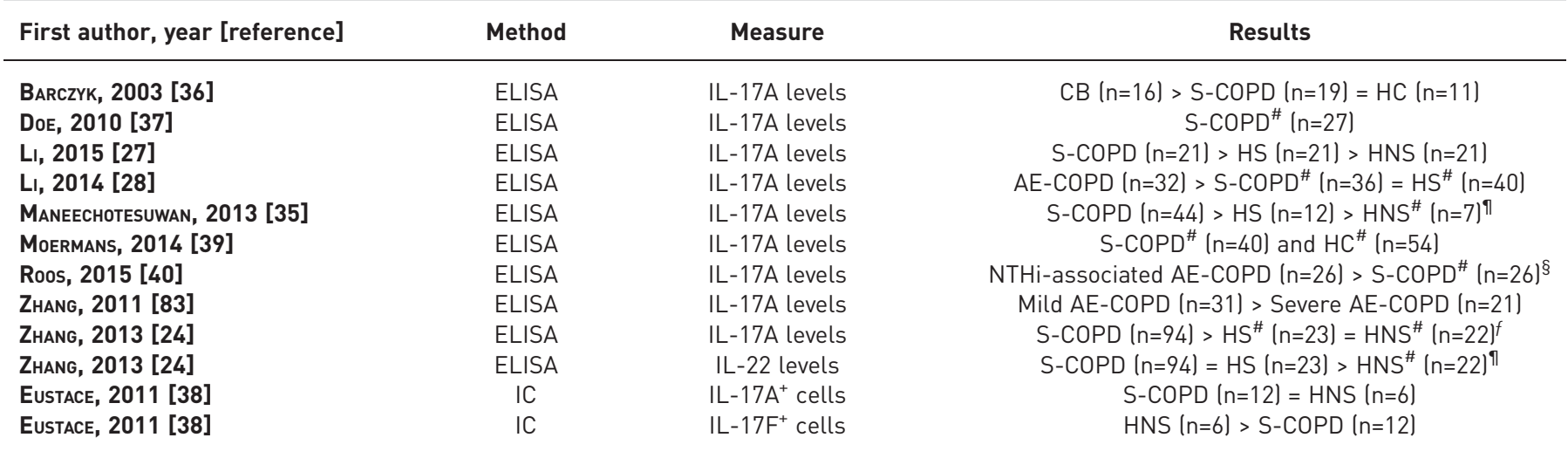

Data are presented as comparisons of cytokine levels or cell proportions in the sputum of healthy nonsmokers (HNS), healthy smokers (HS) and patients with chronic COPD. CB: chronic bronchitis without obstruction; S-COPD: stable COPD; HC: healthy controls without data on smoking status; AE-COPD: acute exacerbation of COPD; NTHi: nontypeable Haemophilus influenzae; IC: immunocytochemical analysis. ${ }^{\#}$ : undetectable level of cytokine; ๆ: cytokine levels increase with COPD stages; ${ }^{\S}$ : sputum collected from the same patients at baseline, during and after AE-COPD; ${ }^{f}$ : IL-17A levels below the lower limit of detection for mild and moderate COPD.

IL-17F-positive cells are present throughout the follicles, suggesting different roles for IL-17A and IL-17F related to the differentiation of $\mathrm{T}$ - and $\mathrm{B}$-cells, respectively $[38,45]$. As shown in an experimental model of rheumatoid arthritis, IL-23-activated Th17 cells located in germinal centres of secondary lymphoid organs are involved in a shift toward pro-inflammatory autoantibodies and trigger the onset of arthritis. This suggests that Th17 cells may be involved in the autoimmune features observed in COPD [50, 51]. Indeed,

TABLE 3 Main results of studies focusing on interleukin (IL)-17- and IL-22-producing cells in lung biopsies of chronic obstructive pulmonary disease (COPD) patients

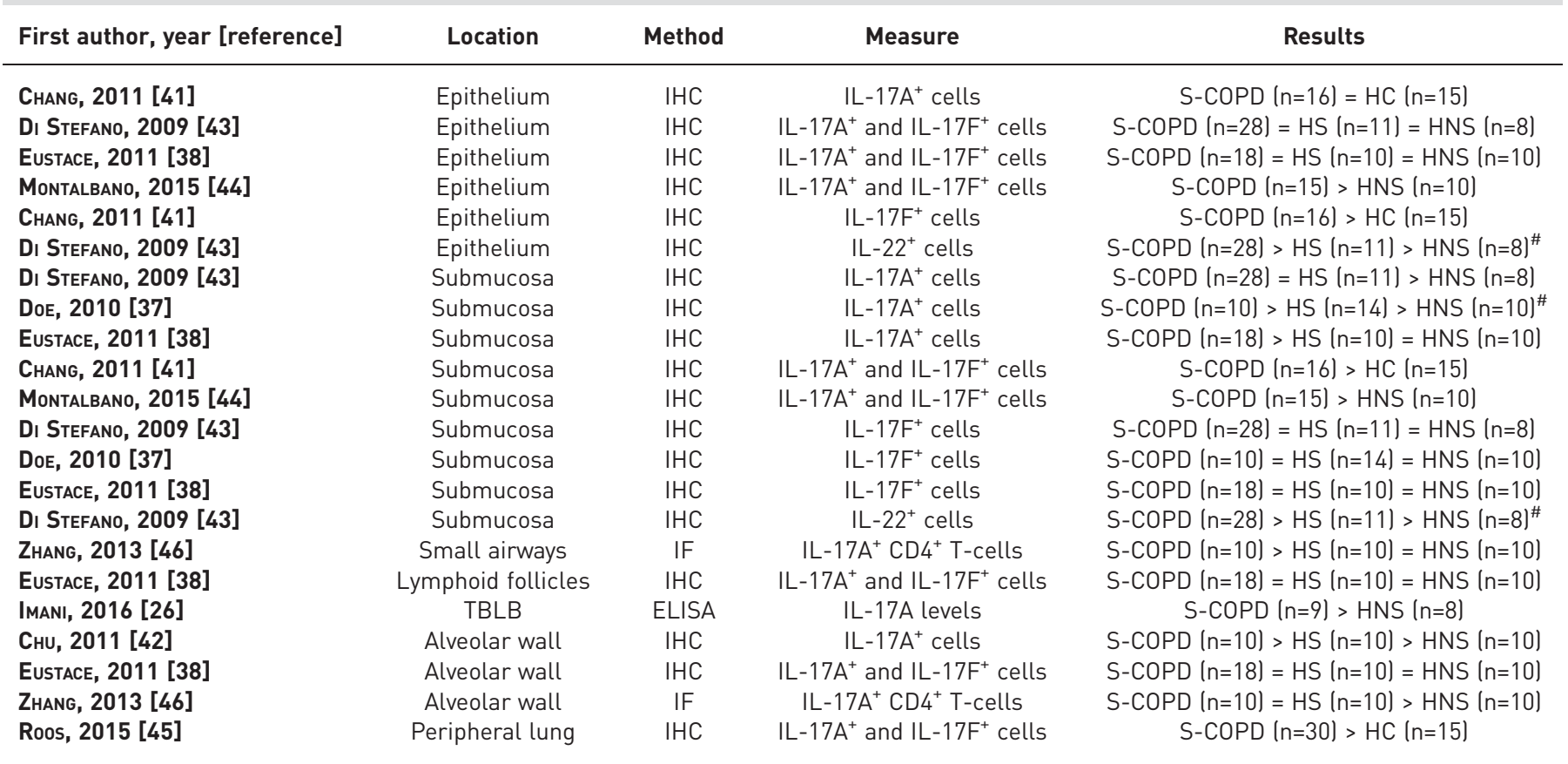

Data are presented as comparisons of cytokine levels or cell proportions in lung biopsies of healthy nonsmokers (HNS), healthy smokers (HS) and patients with COPD. IHC: immunohistochemistry; S-COPD: stable COPD; HC: healthy controls without data on smoking status; IF: immunofluorescence staining; TBLB: transbronchial lung biopsy. ${ }^{\#}$ : S-COPD is significantly different from HNS, but HS is not significantly different from the two other groups. 
the detection of autoantibodies, for instance anti-elastin and anti-cytokeratin 18 antibodies, has been reported, although their pathophysiological role is not well defined $[52,53]$.

In murine models, cigarette smoke induces expansion of Th17 and Tc17 cells, but also increases IL-17A production by nonconventional lymphocytes including NK cells, NKT and $\gamma \delta$ T-cells [54-56]. This production of IL-17A is important, as cigarette smoke-induced inflammation is reduced in $i l 17 a^{-/-}$mice or when using anti-IL-17A neutralising antibodies [55]. Interestingly, NKT have been reported to be recruited and more activated in the airways and in the blood of COPD patients $[56,57]$. In our model of chronically exposed mice, animals deficient in NKT exhibited strongly reduced inflammation and lung function alteration at least during the first step of the exposure to cigarette smoke [56]. Similar findings have been suggested for $\gamma \delta \mathrm{T}$-cells, which are another important cell source for IL-17A [55]. In a model of chronic lung inflammation mimicking COPD features, host-microbiota dialogue promotes inflammation and lung function alteration by inducing IL-17A production [49]. This production, mainly due to Th17 and $\gamma \delta \mathrm{T}$-cells, is induced by changes in airway microbiota. This strongly suggests the potential implication of airway dysbiosis in the promotion of Th17 cytokines in COPD patients. Therefore, IL-17A production by innate immune cells seems to play a key role in the initiation of the disease.

The production of IL-17A is controlled by regulatory cytokines, including IL-10 and TGF- $\beta$ [58]. Interestingly, sputum IL-10 concentrations are lower in COPD patients and negatively correlated to airways obstruction $[24,35]$. This change in the balance between IL-10 and IL-17A was shown to be related to a decreased activity of indoleamine 2,3-dioxygenase, a tolerance inducer with abilities to control oxidative stress in COPD patients [35]. Conversely, some authors have reported an increased concentration of IL-10 and TGF- $\beta$ in plasma of COPD patients associated with an increased regulatory activity in circulating T-cells [59]. In our hands, in vivo exposure to cigarette smoke induced IL-10 expression in the lung tissues [60]. Moreover, $i l 10^{-/}$mice exposed to cigarette smoke have a higher IL-17A response associated with a more severe alteration of lung function. Therefore, these data suggest that in COPD, the IL-10-mediated regulatory response is defective or inadequate to control the Th17-dependent chronic inflammation.

Altogether, these data suggest that COPD pathophysiology may differ between early and later phases of the disease. Indeed, in our experimental model, we observed two waves of Th17 cytokine production: the first related to the activation of innate immune cells and the second associated with the development of Th17 cells. Therefore, we suggest that innate immune mechanisms may occur during the onset of the disease (in response to the first exposure to cigarette smoke), while adaptive immunity may predominate during the later phase of COPD. This might also correspond to different mechanisms of production, since innate lymphocytes are quickly activated by airway epithelial cells and dendritic cells exposed to cigarette smoke. In contrast, the development of Th17 cells requires a more complex process probably involving an alteration of regulatory mechanisms, which may facilitate the development of autoimmune responses.

\section{Roles of IL-17 and IL-22 in airway obstruction}

There is no direct evidence for IL-17A implication in airway obstruction observed in COPD patients, but most clinical studies report significantly higher number of Th17 cells and cytokine levels in COPD patients compared to controls, and some of them a significant inverse correlation with FEV1 [23-26, 29, $32,33,35]$. Evaluation of airway obstruction in murine experimental models is more complex, as measurement of maximum expiratory flows is very difficult. As previous clinical studies have shown that airways responsiveness to inhaled metacholine is frequent in COPD patients and correlates with airways obstruction [61-64], authors use the measurement of resistance after metacholine inhalation as a surrogate for FEV1 in murine models to assess airways obstruction. Interestingly, different murine models have shown that airway hyperreactivity development is IL-17A-dependent [65-67]. Moreover, in a model of mice chronically exposed to cigarette smoke which develop histological and immunological features close to those observed in COPD patients, we confirmed the involvement of IL-17A produced by invariant NKT, a subtype of NKT, in airway hyperreactivity [56]. In another model of mice exposed to 3 days of cigarette smoke, injection of recombinant IL-22 alone or in association with cigarette smoke induces airway hyperreactivity with increased IL-17A and neutrophils, demonstrating possible deleterious effects of this cytokine [68]. Finally, mouse exposure to chlorine induces a neutrophil-dependent airway hyperreactivity [69]. Interestingly, a positive correlation between IL-17A concentrations and neutrophil counts was also observed in sputum of COPD patients [24]. These results are strengthened by a recent study showing that IL-17A is essential for small airway fibrosis and inflammation in mice exposed to cigarette smoke, suggesting a role for this cytokine in airway obstruction during COPD [70, 71]. Altogether, these experimental data suggest that Th17 cytokines produced by immune cells in inflamed lungs are involved in airway obstruction development through neutrophil recruitment, a context reproducing COPD. 
Role of IL-17 and IL-22 in emphysema

Emphysema is the consequence of alveolar wall destruction, which is a common feature of COPD [71]. Mice exposed to cigarette smoke for 6 months develop airspace enlargement associated with increased CCR $^{+}$Th17 cells [72]. Moreover, $c c r 6^{-1-}$ mice are protected against cigarette smoke-induced emphysema, suggesting a pathogenic role of these Th17 cells [73]. Molecular mechanisms of cigarette smoke-induced Th17 differentiation and activation have not been fully clarified, but it was shown in vitro that this differentiation is AHR-dependent [74]. However, in addition, inflammasome activation seems to be important, as IL-17A induction is reduced in $i l 1 r 1^{-/-}$and $n l r p 3^{-/-}$mice $[40,66]$. Finally, cigarette smoke-induced microRNA (miR)-22 expression in APCs is also required to promote Th17 cell-dependent emphysema, demonstrating that different pathways may be involved [75].

Interestingly, IL-17RA signalling is required for emphysema development [74]. Moreover, $i l 17 a^{-/-}$and $i l 23^{-1-}$ mice are protected against elastase-induced emphysema, an effect associated with lower levels of neutrophil chemotactic factors keratinocyte chemoattractant and macrophage inflammatory protein-2 (CXCL1 and CXCL2) and lower neutrophil numbers, demonstrating that the development of emphysema is controlled by the IL-23/IL-17A pathway $[76,77]$. In this model, combination of IL-17A and IL-22 is able to increase the expression of matrix metalloproteinases, molecules involved in tissue destruction [71]. Furthermore, $i l-17 a^{-/-}$mice and wild-type mice treated with neutralising anti-IL-17A antibody acutely exposed to cigarette smoke exhibit a lower macrophage and neutrophil recruitment associated with a reduced mRNA expression of chemokine (C-C motif) ligand 2 (CCL2), CCL3 and matrix metalloproteinase-12 [55]. These data suggest that IL-17A and IL-22 induce emphysema via proteases release by immune and epithelial cells [71, 78]. Finally, cigarette smoke-induced IL-17A directly contributes to type II alveolar cell apoptosis [79]. Therefore, IL-17A and possibly IL-22 promote emphysema through production of proteases and induction of alveolar cell apoptosis.

\section{IL-17 and IL-22 in COPD exacerbations}

Under normal conditions without chronic inflammation, IL-17A and IL-22 are key factors controlling extracellular bacterial and fungal infections [80-82]. In addition, these cytokines are important in COPD exacerbations, but their involvement seems to be more complex and may depend on the pathogen responsible. COPD patients with airway colonisation by opportunistic pathogens exhibit lower blood IL-17A concentrations at steady state and undetectable level during exacerbations [20]. Moreover, COPD severity is correlated with decreased sputum IL-17A concentrations during exacerbation [83]. However, these results need to be confirmed with larger cohorts and it seems that the aetiology of the exacerbation should be taken in to account.

Nontypeable Haemophilus influenzae (NTHi) are the most common bacteria associated with COPD exacerbation [3]. NTHi-associated COPD exacerbations are associated with increased sputum IL-17A concentration [40]. Despite decreased neutrophil recruitment in $i l 17 a^{-/-}$mice and in wild-type mice treated with an anti-IL-17A neutralising antibody, no increased bacterial burden or delayed bacterial clearance were observed, showing that IL-17A was not involved in the clearance of NTHi during COPD [40]. In contrast, during infection by $\mathrm{NTHi}$, we demonstrated a protective effect of IL-22 which is decreased in COPD mice compared to mice not exposed to cigarette smoke [60]. Streptococcus pneumoniae is another bacteria frequently associated with COPD exacerbations [3]. IL-17A and IL-22 were shown to be involved in the protective host response against this bacteria $[80,81,84]$. In mice chronically exposed to cigarette smoke, we reported a defective IL-17A and IL-22 response to S. pneumoniae, which was related to reduced production of IL-1 $\beta$ and IL-23 by alveolar macrophages and dendritic cells [85]. In addition, we observed that in vitro exposure to cigarette smoke altered the response of dendritic cells to $S$. pneumoniae, leading to a decreased ability to promote Th17 differentiation of T-cells [86]. Ex vivo exposure to $S$. pneumoniae of peripheral blood mononuclear cells from COPD patients does not increase IL-17A and IL-22 production, in contrast to healthy nonsmokers and healthy smokers [85]. In contrast, the phenotype of lung dendritic cells is modified similarly in COPD patients and healthy smokers [87]. These data suggest that defective systemic response to bacteria is a specific feature of COPD that may be the consequence of the conjugate effect of exposure to cigarette smoke, systemic chronic inflammation associated with the disease and/or host predisposition to develop the disease. Altogether, these data suggest that impaired balance between IL-17A and IL-22 with a defective production of the latter is a main feature in COPD, leading to bacterial infection and colonisation of the airways (figure 2).

Human influenza virus and rhinovirus (HRV) are also associated with COPD exacerbations [3]. In wild-type mice not exposed to cigarette smoke, influenza virus induces production of IL-17A and IL-22 by conventional T-cells and innate lymphoid cells with IL-22-dependent protective effects against secondary bacterial infection [81, 88, 89]. Most of these effects of IL-22 are related to the maintenance of the epithelial barrier and the limitation of airway remodelling. However, we cannot exclude that this cytokine 
acts by promoting the production of antimicrobial peptides. Conversely, mice chronically exposed to cigarette smoke exhibit an alteration of IL-22-dependent signalling during influenza virus infection [90]. Influenza virus induces IL-22R expression on bronchial epithelial cells which is cleaved by neutrophil proteases leading to decreased production of antimicrobial peptides (figure 2). Levels of soluble fragments

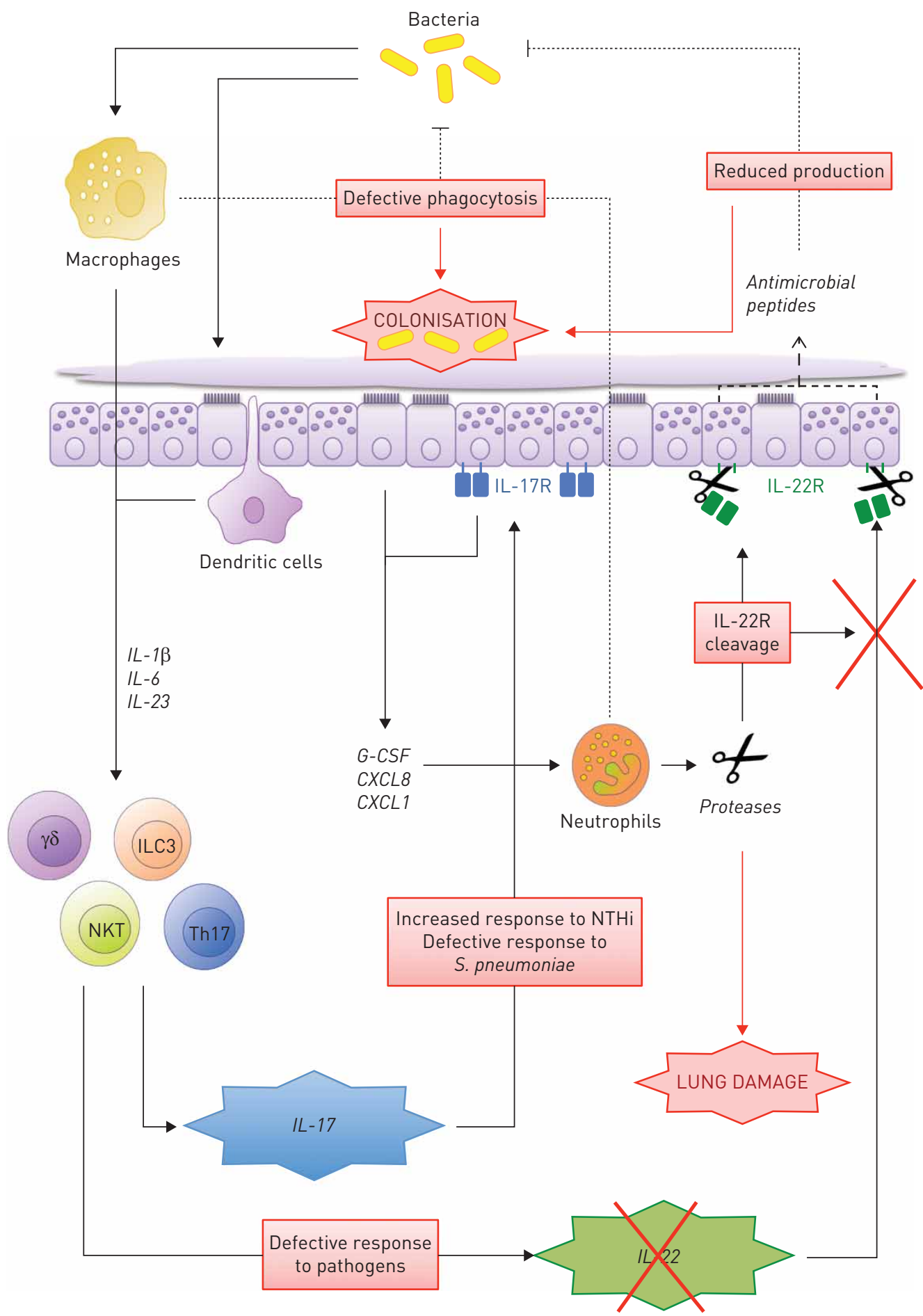

FIGURE 2 Defective interleukin (IL)-22 and differential IL-17 responses during chronic obstructive pulmonary disease (COPD) exacerbations. $\gamma \delta$ : $\gamma \delta$ T-cell; ILC3: innate lymphoid cell type 3; NKT: natural killer T-cell; Th17: T-helper type 17; G-CSF: granulocyte colony-stimulating factor; CXCL8: IL-8; CXCL1: chemokine C-X-C motif ligand 1; IL-17R: IL-17 receptor; IL-22R: IL-22 receptor; NTHi: nontypeable Haemophilus influenzae; S. pneumoniae: Streptococcus pneumoniae. 
of IL-22R are increased in sputa from COPD patients with acute exacerbations, thus confirming the alteration of this pathway in COPD patients [90]. HRVs are the most frequent viruses associated with COPD exacerbation [3]. IL-17A synergistically enhances HRV-16-induced production of CXCL8 and $\beta$-defensin-2 and suppresses production of CCL5 [91]. Experimental HRV infection induces an exacerbation associated with a secondary bacterial infection in $60 \%$ of COPD patients, compared with $9.5 \%$ in healthy smokers and $10 \%$ in healthy nonsmokers [92]. Interestingly, antimicrobial peptide concentration is lower in sputa from these COPD patients during HRV-induced exacerbation compared with control individuals and these levels are negatively correlated with bacterial load [92]. Thus, in COPD, viral infections may lead to increased inflammation and symptoms in the context of pre-existing high levels of IL-17A. This may be associated with increased risk of subsequent bacterial infection due to altered antimicrobial peptide response to IL-17A and/or IL-22.

Although COPD patients are known to be at risk of invasive or chronic necrotising aspergillosis, no data are available on the role of Th17 response in fungal infections in the context of COPD [93, 94]. The presence of Aspergillus-specific T-cells exhibiting a Th17 phenotype and producing IL-17A has been demonstrated recently in the lungs of COPD patients, whereas Aspergillus-specific T-cells showed a Th1 phenotype in the peripheral blood [95]. Aspergillus fumigatus, the main species responsible for these infections, has been shown to induce IL-17A, IL-17F and IL-22 secretion through the dectin-1-dependent production of IL-1 $\beta$, IL-6 and IL-23 [96]. A defective expression of dectin-1 was found in individuals with haematological malignancies who developed invasive aspergillosis when compared to patients who had no evidence of invasive fungal infection [97]. However, the role for Th17 response in aspergillosis remains controversial, with data obtained from different mouse models suggesting it could be pathogenic [98, 99]. Therefore, further studies in a COPD context are needed to support the hypothesis that defective Th17 response during the course of COPD might facilitate the development of fungal infections.

\section{Future directions}

Cellular and molecular mechanisms underlying COPD are poorly understood and current pharmacotherapy is rather ineffective. The picture becomes more complex when we consider the heterogeneous phenotype of COPD patients, in terms of stage and progression of the disease, comorbidities, etc. Thus, there are currently no drugs that significantly reduce disease progression or mortality and prevent COPD exacerbations. In recent years, the most important advance in the management of the disease has been the introduction of long-acting bronchodilators. However, those drugs do not lower the underlying chronic inflammatory response that occurs in the lung mucosa of COPD patients. Moreover, inflammation in COPD lungs is essentially resistant to broad-spectrum anti-inflammatory molecules such as corticosteroids. Thus, there is an obvious need for anti-inflammatory therapies that can act on new promising molecular and/or cellular targets [100]. Among those, Th17 cytokines appear to play important but opposite roles during both stable and exacerbation phases of COPD. Increases in IL-17A seems to be more related to chronic inflammation leading to airway obstruction and emphysema, whereas defects in IL-22 seem to be responsible for the lack of control of infection leading to exacerbations and colonisation of the airways. Nevertheless, because COPD is associated to a chronic and deleterious lung inflammatory process, it seems essential to decipher the cytokine-specific mechanisms implicated in these alterations.

As underlined above, the production of IL-17A implicates different cell sources and mechanisms of activation, including oxidative stress. Direct activation of APCs (including airway epithelial cells) by cigarette smoke can lead to activation of NKTs, a process blocked by the preventive administration of antioxidants [56]. Nevertheless, clinical trials evaluating the effects of the antioxidant $\mathrm{N}$-acetylcysteine in COPD showed mixed results with significant reductions in exacerbation frequency in Asian population, but no clear changes in disease progression, emphasising the need for new therapeutic targets [101]. Therefore, blocking IL-17A production seems to be a promising therapeutic target in COPD patients, as reported in a mouse model with a reduction of lung damage without diminishing control of bacterial infections [40]. However, these clinical trials had a preventive design and additional studies are still needed to evaluate the benefits of inhibition of the IL-17A pathway when the disease is already established. Moreover, clinical data from patients with psoriasis and treated with secukinumab, an anti-IL-17A antibody, showed higher rates of infection, suggesting that this treatment may be associated with more exacerbations in the context of COPD [102]. In view of the marked heterogeneity of the inflammatory response and clinical phenotype in COPD patients, it may be more appropriate to target the cell signalling pathways regulating IL-17A expression (i.e. ROR $\gamma \mathrm{t}$, peroxisome proliferator-activated receptor- $\gamma$ and kynurenine metabolism) rather than blocking this class of cytokines per se or its receptor [103]. However, this approach should be evaluated carefully, as preclinical studies in mouse models have shown high risk of lymphoma if ROR $\gamma \mathrm{t}$ is completely repressed [104]. Treatment with simvastatin (a 3-hydroxy-3-methyl-glutarylcoenzyme-A reductase inhibitor) in stable COPD patients decreased the levels 
of both IL-17A and IL-22 in the sputum, whereas it increased IL-10 production [105]. However, this is not associated with an improvement of clinical outcomes after a short period of treatment. Hence, further studies are needed to thoroughly evaluate the risk-benefit ratio of inhibiting the IL-17A pathway in patients with COPD.

During infection, the defect in IL-22 production associated with the defective APC response (particularly alveolar macrophages and dendritic cells) seems to be a key feature of exacerbation. However, we cannot exclude that this mechanism may also be associated with a dysfunction of lymphocytes, as suggested in our model with NTHi [60]. Deciphering the mechanism responsible for the altered production and function of IL-22 seems essential in order to propose a therapeutic approach in this context. Since alteration of the function of dendritic cells by cigarette smoke is not mainly dependent on oxidative stress, we suspect that exposure to cigarette smoke alters phagolysosome trafficking, which secondarily impacts the signalling pathways triggered by bacteria [86]. In addition, dysregulation of miRNA expression after cigarette smoke exposure is implicated in dendritic cell dysfunction, although their role during exacerbations has not yet been evaluated [75]. Interestingly, our data showed that IL-22 supplementation increases bacterial clearance and markedly reduces lung inflammation and tissue lesions, underlining interest in an immunotherapeutic approach aiming to boost this response to bacteria [60, 85]. The administration of this cytokine is presently being evaluated in alcoholic hepatitis and in lower gastrointestinal acute graft-versus-host disease in haematopoietic stem cell transplantation recipients (ClinicalTrials.gov: NCT02655510, NCT02406651). Based on our experiments, two strategies might be planned: either to boost IL-22-producing cells with immunostimulants and/or to amplify the function of this cytokine. Some adjuvants such as flagellin have the ability to promote the IL-22 response through the stimulation of the dendritic cell-ILC3 axis and thus promote the clearance of bacteria in mice [84]. Administration of this adjuvant in association with other treatments of COPD exacerbations may be of great interest, as it would both improve local IL-22 production in the infection site and reduce systemic side-effects. As the function of IL-22 is altered due to the specific cleavage of its receptor by neutrophil proteases, specific antineutrophil proteases might have a double therapeutic interest, i.e. not only by protecting the IL-22R on airway epithelium, but also by limiting the degradation of the extracellular matrix and the destruction of alveolar wall [90]. Another important effect might be related to the effect of Th17 cytokines on the composition and the quality of the epithelial lining fluid. Indeed, these cytokines might promote the production of mucins such as MUC5B, which have important antibacterial effects [106]. Moreover, restoring a neutral $\mathrm{pH}$ in the lining fluid of COPD patients through cystic fibrosis transmembrane conductance regulator activation by IL-17A might help to clear bacteria as shown in the context of cystic fibrosis [13]. Altogether, this might restore an efficient antimicrobial response (including the production of antibacterial peptides) by airway epithelium, and might limit tissue lesions and the progression of the disease associated with exacerbation.

Similar to asthma, identifying different COPD endotypes might be useful to fully guide future therapeutics. COPD pathophysiology is characterised by neutrophilic inflammation associated with Th17 response [71, 107]. Whereas the severity of some COPD patients is characterised by the presence of eosinophilic inflammation, to our knowledge there is always associated neutrophilic inflammation [108], a process related to the effect of IL-17A. In addition, only a subgroup of COPD patients exhibit frequent exacerbations [109]. Recent data showed that an eosinophilic endotype may be associated with higher risk of exacerbations [110]. This suggests that frequent exacerbators among COPD patients may be characterised by different biological endotypes [111]. Therefore, biomarker identification to predict the risk and the frequency of future exacerbations would be of great interest. Interestingly, we found defective IL-22 production by bacteria-activated blood mononuclear cells from COPD patients [85]. This in vitro test might help to identify COPD patients susceptible to developing pathogen-associated exacerbation and to identify patients that need preventive immunostimulatory treatment including vaccination.

\section{Conclusion}

On the whole, it seems essential to better define the complex cellular network controlling the production and the function of Th17 cytokines in COPD in order to propose efficient therapeutic approaches. Our present knowledge underlines the interest in different therapeutic or preventive approaches targeting the development of COPD, as well as exacerbations, by carefully modulating the balance between IL-17A and IL-22, and their receptors. These cytokines and their related molecules might be used as markers of disease progression and might be helpful for the management of COPD patients and their treatment. 
Contributors: all authors conceived and designed the review. O. Le Rouzic and P. Gosset made the selection of the articles and designed the figures. M. Pichavant has worked on the IL-17 contribution in bronchial obstruction and has contributed to define future directions. E. Frealle has conducted research concerning the implication of Th17 cytokines and fungal infections during COPD. A. Guillon and M. Si-Tahar wrote the section on cytokine receptors and have contributed to the future directions. All authors critically revised the manuscript, and gave final approval of this version to be published.

\section{References}

1 Global Initiative for Chronic Obstructive Lung Disease. GOLD 2017 Global Strategy for the Diagnosis, Management and Prevention of COPD. http://goldcopd.org. Date last accessed: June 1, 2017. Date last updated: January 1, 2017.

2 Adeloye D, Chua S, Lee C, et al. Global and regional estimates of COPD prevalence: systematic review and meta-analysis. J Glob Health 2015; 5: 020415.

3 Sethi S, Murphy TF. Infection in the pathogenesis and course of chronic obstructive pulmonary disease. $N$ Engl $J$ Med 2008; 359: 2355-2365.

4 Pavord ID, Jones PW, Burgel P-R, et al. Exacerbations of COPD. Int J Chron Obstruct Pulmon Dis 2016; 11: 21-30.

5 Dransfield MT, Kunisaki KM, Strand MJ, et al. Acute exacerbations and lung function loss in smokers with and without chronic obstructive pulmonary disease. Am J Respir Crit Care Med 2017; 195: 324-330.

6 McAleer JP, Kolls JK. Directing traffic: IL-17 and IL-22 coordinate pulmonary immune defense. Immunol Rev 2014; 260: 129-144.

$7 \quad$ Rathore JS, Wang Y. Protective role of Th17 cells in pulmonary infection. Vaccine 2016; 34: 1504-1514.

8 Iwasaki A, Foxman EF, Molony RD. Early local immune defences in the respiratory tract. Nat Rev Immunol 2017; 17: 7-20.

9 Pappu R, Rutz S, Ouyang W. Regulation of epithelial immunity by IL-17 family cytokines. Trends Immunol 2012; 33: 343-349.

10 Rutz S, Eidenschenk C, Ouyang W. IL-22, not simply a Th17 cytokine. Immunol Rev 2013; 252: 116-132.

11 Sabat R, Ouyang W, Wolk K. Therapeutic opportunities of the IL-22-IL-22R1 system. Nat Rev Drug Discov 2014; 13: 21-38.

12 Kreindler JL, Bertrand CA, Lee RJ, et al. Interleukin-17A induces bicarbonate secretion in normal human bronchial epithelial cells. Am J Physiol Lung Cell Mol Physiol 2009; 296: L257-L266.

13 Pezzulo AA, Tang XX, Hoegger MJ, et al. Reduced airway surface $\mathrm{pH}$ impairs bacterial killing in the porcine cystic fibrosis lung. Nature 2012; 487: 109-113.

14 Liang SC, Long AJ, Bennett F, et al. An IL-17F/A heterodimer protein is produced by mouse Th17 cells and induces airway neutrophil recruitment. J Immunol 2007; 179: 7791-7799.

15 Aujla SJ, Chan YR, Zheng M, et al. IL-22 mediates mucosal host defense against Gram-negative bacterial pneumonia. Nat Med 2008; 14: 275-281.

16 Sonnenberg GF, Nair MG, Kirn TJ, et al. Pathological versus protective functions of IL-22 in airway inflammation are regulated by IL-17A. J Exp Med 2010; 207: 1293-1305.

17 Patel DD, Kuchroo VK. Th17 cell pathway in human immunity: lessons from genetics and therapeutic interventions. Immunity 2015; 43: 1040-1051.

18 McGeachy MJ, Bak-Jensen KS, Chen Y, et al. TGF- $\beta$ and IL-6 drive the production of IL-17 and IL-10 by T cells and restrain TH-17 cell-mediated pathology. Nat Immunol 2007; 8: 1390-1397.

19 Lee Y, Awasthi A, Yosef N, et al. Induction and molecular signature of pathogenic TH17 cells. Nat Immunol 2012; 13: 991-999.

20 Andelid K, Tengvall S, Andersson A, et al. Systemic cytokine signaling via IL-17 in smokers with obstructive pulmonary disease: a link to bacterial colonization? Int J Chron Obstruct Pulmon Dis 2015; 10: 689-702.

21 Jin Y, Wan Y, Chen G, et al. Treg/IL-17 ratio and Treg differentiation in patients with COPD. PLoS One 2014; 9 ; e111044.

22 Sun N, Wei X, Wang J, et al. Caveolin-1 promotes the imbalance of Th17/Treg in patients with chronic obstructive pulmonary disease. Inflammation 2016; 39: 2008-2015.

23 Wang H, Ying H, Wang S, et al. Imbalance of peripheral blood Th17 and Treg responses in patients with chronic obstructive pulmonary disease. Clin Respir J 2015; 9: 330-341.

24 Zhang L, Cheng Z, Liu W, et al. Expression of interleukin (IL)-10, IL-17A and IL-22 in serum and sputum of stable chronic obstructive pulmonary disease patients. COPD 2013; 10: 459-465.

25 Zou Y, Chen X, Liu J, et al. Serum IL-1 $\beta$ and IL-17 levels in patients with COPD: associations with clinical parameters. Int J Chron Obstruct Pulmon Dis 2017; 12: 1247-1254.

26 Imani S, Salimian J, Fu J, et al. Th17/Treg-related cytokine imbalance in sulfur mustard exposed and stable chronic obstructive pulmonary (COPD) patients: correlation with disease activity. Immunopharmacol Immunotoxicol 2016; 38: 270-280.

27 Li H, Liu Q, Jiang Y, et al. Disruption of Th17/Treg balance in the sputum of patients with chronic obstructive pulmonary disease. Am J Med Sci 2015; 349: 392-397.

28 Li X-N, Pan X, Qiu D. Imbalances of Th17 and Treg cells and their respective cytokines in COPD patients by disease stage. Int J Clin Exp Med 2014; 7: 5324-5329.

29 Paats MS, Bergen IM, Hoogsteden HC, et al. Systemic $\mathrm{CD}^{+}$and $\mathrm{CD} 8^{+}$T-cell cytokine profiles correlate with GOLD stage in stable COPD. Eur Respir J 2012; 40: 330-337.

30 Qiu S-L, Duan M-C, Liang Y, et al. Cigarette smoke induction of interleukin-27/WSX-1 regulates the differentiation of Th1 and Th17 cells in a smoking mouse model of emphysema. Front Immunol 2016; 7: 553.

31 Solleiro-Villavicencio H, Quintana-Carrillo R, Falfán-Valencia R, et al. Chronic obstructive pulmonary disease induced by exposure to biomass smoke is associated with a Th2 cytokine production profile. Clin Immunol 2015; 161: $150-155$

32 Vargas-Rojas MI, Ramírez-Venegas A, Limón-Camacho L, et al. Increase of Th17 cells in peripheral blood of patients with chronic obstructive pulmonary disease. Respir Med 2011; 105: 1648-1654. 

lung function in patients with chronic obstructive pulmonary disease. Chin Med J 2016; 129: 909-916.

34 Zhang J-C, Chen G, Chen L, et al. TGF- $3 /$ BAMBI pathway dysfunction contributes to peripheral Th17/Treg imbalance in chronic obstructive pulmonary disease. Sci Rep 2016; 6: 31911.

35 Maneechotesuwan K, Kasetsinsombat K, Wongkajornsilp A, et al. Decreased indoleamine 2,3-dioxygenase activity and IL-10/IL-17A ratio in patients with COPD. Thorax 2013; 68: 330-337.

36 Barczyk A, Pierzchala W, Sozañska E. Interleukin-17 in sputum correlates with airway hyperresponsiveness to methacholine. Respir Med 2003; 97: 726-733.

37 Doe C, Bafadhel M, Siddiqui S, et al. Expression of the T helper 17-associated cytokines IL-17A and IL-17F in asthma and COPD. Chest 2010; 138: 1140-1147.

38 Eustace A, Smyth LJC, Mitchell L, et al. Identification of cells expressing IL-17A and IL-17F in the lungs of patients with COPD. Chest 2011; 139: 1089-1100.

39 Moermans C, Bonnet C, Willems E, et al. Sputum cytokine levels in patients undergoing hematopoietic SCT and comparison with healthy subjects and COPD: a pilot study. Bone Marrow Transplant 2014; 49: 1382-1388.

40 Roos AB, Sethi S, Nikota J, et al. IL-17A and the promotion of neutrophilia in acute exacerbation of chronic obstructive pulmonary disease. Am J Respir Crit Care Med 2015; 192: 428-437.

41 Chang Y, Nadigel J, Boulais N, et al. CD8 positive T cells express IL-17 in patients with chronic obstructive pulmonary disease. Respir Res 2011; 12: 43.

42 Chu S, Zhong X, Zhang J, et al. The expression of Foxp3 and ROR gamma $\mathrm{t}$ in lung tissues from normal smokers and chronic obstructive pulmonary disease patients. Int Immunopharmacol 2011; 11: 1780-1788.

43 Di Stefano A, Caramori G, Gnemmi I, et al. T helper type 17-related cytokine expression is increased in the bronchial mucosa of stable chronic obstructive pulmonary disease patients. Clin Exp Immunol 2009; 157: 316-324.

44 Montalbano AM, Riccobono L, Siena L, et al. Cigarette smoke affects IL-17A, IL-17F and IL-17 receptor expression in the lung tissue: ex vivo and in vitro studies. Cytokine 2015; 76: 391-402.

45 Roos AB, Sandén C, Mori M, et al. IL-17A is elevated in end-stage chronic obstructive pulmonary disease and contributes to cigarette smoke-induced lymphoid neogenesis. Am J Respir Crit Care Med 2015; 191: $1232-1241$.

46 Zhang J, Chu S, Zhong X, et al. Increased expression of CD $4^{+} \mathrm{IL}-17^{+}$cells in the lung tissue of patients with stable chronic obstructive pulmonary disease (COPD) and smokers. Int Immunopharmacol 2013; 15 : 58-66.

47 Hogg JC, Chu F, Utokaparch S, et al. The nature of small-airway obstruction in chronic obstructive pulmonary disease. N Engl J Med 2004; 350: 2645-2653.

48 Hwang JY, Randall TD, Silva-Sanchez A. Inducible bronchus-associated lymphoid tissue: taming inflammation in the lung. Front Immunol 2016; 7: 258

49 Yadava K, Pattaroni C, Sichelstiel AK, et al. Microbiota promotes chronic pulmonary inflammation by enhancing il-17a and autoantibodies. Am J Respir Crit Care Med 2016; 193: 975-987.

50 Pfeifle R, Rothe T, Ipseiz N, et al. Regulation of autoantibody activity by the IL-23-TH17 axis determines the onset of autoimmune disease. Nat Immunol 2017; 18: 104-113.

51 Bagdonas E, Raudoniute J, Bruzauskaite I, et al. Novel aspects of pathogenesis and regeneration mechanisms in COPD. Int J Chron Obstruct Pulmon Dis 2015; 10: 995-1013.

52 Lee S-H, Goswami S, Grudo A, et al. Antielastin autoimmunity in tobacco smoking-induced emphysema. Nat Med 2007; 13: 567-569.

53 Kuo Y-B, Chang CA, Wu Y-K, et al. Identification and clinical association of anti-cytokeratin 18 autoantibody in COPD. Immunol Lett 2010; 128: 131-136.

54 Zhou H, Hua W, Jin Y, et al. Tc17 cells are associated with cigarette smoke-induced lung inflammation and emphysema. Respirology 2015; 20: 426-433.

55 Bozinovski S, Seow HJ, Chan SPJ, et al. Innate cellular sources of interleukin-17A regulate macrophage accumulation in cigarette-smoke-induced lung inflammation in mice. Clin Sci 2015; 129: 785-796.

56 Pichavant M, Rémy G, Bekaert S, et al. Oxidative stress-mediated iNKT-cell activation is involved in COPD pathogenesis. Mucosal Immunol 2014; 7: 568-578.

57 Wang J, Urbanowicz RA, Tighe PJ, et al. Differential activation of killer cells in the circulation and the lung: a study of current smoking status and chronic obstructive pulmonary disease (COPD). PLoS One 2013; 8: e58556.

58 Korn T, Bettelli E, Oukka M, et al. IL-17 and Th17 cells. Annu Rev Immunol 2009; 27: 485-517.

59 Kalathil SG, Lugade AA, Pradhan V, et al. T-regulatory cells and programmed death $1^{+} \mathrm{T}$ cells contribute to effector T-cell dysfunction in patients with chronic obstructive pulmonary disease. Am J Respir Crit Care Med 2014; 190: 40-50.

60 Sharan R, Perez-Cruz M, Kervoaze G, et al. Interleukin-22 protects against non-typeable Haemophilus influenzae infection: alteration during chronic obstructive pulmonary disease. Mucosal Immunol 2017; 10: 139-149.

61 Tashkin DP, Altose MD, Bleecker ER, et al. The Lung Health Study: airway responsiveness to inhaled methacholine in smokers with mild to moderate airflow limitation. Am Rev Respir Dis 1992; 145: 301-310.

62 Wise RA, Kanner RE, Lindgren P, et al. The effect of smoking intervention and an inhaled bronchodilator on airways reactivity in COPD: the Lung Health Study. Chest 2003; 124: 449-458.

63 Hassan NM, Hargreave FE, Nair P. Airway responsiveness to indirect challenges in COPD. COPD 2010; 7: $133-140$.

64 van den Berge M, Vonk JM, Gosman M, et al. Clinical and inflammatory determinants of bronchial hyperresponsiveness in COPD. Eur Respir J 2012; 40: 1098-1105.

65 Kim HY, Lee HJ, Chang Y-J, et al. Interleukin-17-producing innate lymphoid cells and the NLRP3 inflammasome facilitate obesity-associated airway hyperreactivity. Nat Med 2014; 20: 54-61.

66 Pauwels NS, Bracke KR, Dupont LL, et al. Role of IL- $1 \alpha$ and the Nlrp3/caspase-1/IL-1 $\beta$ axis in cigarette smoke-induced pulmonary inflammation and COPD. Eur Respir J 2011; 38: 1019-1028.

67 Pichavant M, Goya S, Meyer EH, et al. Ozone exposure in a mouse model induces airway hyperreactivity that requires the presence of natural killer T cells and IL-17. J Exp Med 2008; 205: 385-393.

68 Li J, Zhou W, Huang K, et al. Interleukin-22 exacerbates airway inflammation induced by short-term exposure to cigarette smoke in mice. Acta Pharmacol Sin 2014; 35: 1393-1401. 
McGovern TK, Goldberger M, Allard B, et al. Neutrophils mediate airway hyperresponsiveness after chlorine-induced airway injury in the mouse. Am J Respir Cell Mol Biol 2015; 52: 513-522.

Yanagisawa H, Hashimoto M, Minagawa S, et al. Role of IL-17A in murine models of COPD airway disease. Am J Physiol Lung Cell Mol Physiol 2017; 312: L122-L130.

Barnes PJ. Immunology of asthma and chronic obstructive pulmonary disease. Nat Rev Immunol 2008; 8: 183-192. Harrison OJ, Foley J, Bolognese BJ, et al. Airway infiltration of $\mathrm{CD}^{+} \mathrm{CCR}^{+}{ }^{+}$Th17 type cells associated with chronic cigarette smoke induced airspace enlargement. Immunol Lett 2008; 121: 13-21.

Bracke KR, D'hulst AI, Maes T, et al. Cigarette smoke-induced pulmonary inflammation and emphysema are attenuated in CCR6-deficient mice. J Immunol 2006; 177: 4350-4359.

Chen K, Pociask DA, McAleer JP, et al. IL-17RA is required for CCL2 expression, macrophage recruitment, and emphysema in response to cigarette smoke. PLoS One 2011; 6: e20333.

$\mathrm{Lu}$ W, You R, Yuan X, et al. The microRNA miR-22 inhibits the histone deacetylase HDAC4 to promote $\mathrm{T}_{\mathrm{H}} 17$ cell-dependent emphysema. Nat Immunol 2015; 16: 1185-1194.

Kurimoto E, Miyahara N, Kanehiro A, et al. IL-17A is essential to the development of elastase-induced pulmonary inflammation and emphysema in mice. Respir Res 2013; 14: 5.

Fujii U, Miyahara N, Taniguchi A, et al. IL-23 is essential for the development of elastase-induced pulmonary inflammation and emphysema. Am J Respir Cell Mol Biol 2016; 55: 697-707.

Overbeek SA, Braber S, Koelink PJ, et al. Cigarette smoke-induced collagen destruction; key to chronic neutrophilic airway inflammation? PLoS One 2013; 8: e55612.

Chang Y, Al-Alwan L, Audusseau S, et al. Genetic deletion of IL-17A reduces cigarette smoke-induced inflammation and alveolar type II cell apoptosis. Am J Physiol Lung Cell Mol Physiol 2014; 306: L132-L143.

Ivanov S, Fontaine J, Paget C, et al. Key role for respiratory $\mathrm{CD}_{103^{+}}$dendritic cells, IFN- $\gamma$, and IL-17 in protection against Streptococcus pneumoniae infection in response to $\alpha$-galactosylceramide. J Infect Dis 2012; 206: 723-734.

Ivanov S, Renneson J, Fontaine J, et al. Interleukin-22 reduces lung inflammation during influenza A virus infection and protects against secondary bacterial infection. J Virol 2013; 87: 6911-6924.

Wozniak KL, Hole CR, Yano J, et al. Characterization of IL-22 and antimicrobial peptide production in mice protected against pulmonary Cryptococcus neoformans infection. Microbiology 2014; 160: 1440-1452.

Zhang $\mathrm{X}$, Zheng $\mathrm{H}$, Zhang $\mathrm{H}$, et al. Increased interleukin (IL)-8 and decreased IL-17 production in chronic obstructive pulmonary disease (COPD) provoked by cigarette smoke. Cytokine 2011; 56: 717-725.

Van Maele L, Carnoy C, Cayet D, et al. Activation of type 3 innate lymphoid cells and interleukin 22 secretion in the lungs during Streptococcus pneumoniae infection. J Infect Dis 2014; 210: 493-503.

Pichavant M, Sharan R, Le Rouzic O, et al. IL-22 defect during Streptococcus pneumoniae infection triggers exacerbation of chronic obstructive pulmonary disease. EBioMedicine 2015; 2: 1686-1696.

Le Rouzic O, Koné B, Kluza J, et al. Cigarette smoke alters the ability of human dendritic cells to promote anti-Streptococcus pneumoniae Th17 response. Respir Res 2016; 17: 94.

Liao S-X, Ding T, Rao X-M, et al. Cigarette smoke affects dendritic cell maturation in the small airways of patients with chronic obstructive pulmonary disease. Mol Med Rep 2015; 11: 219-225.

Sichelstiel A, Yadava K, Trompette A, et al. Targeting IL-1 $\beta$ and IL-17A driven inflammation during influenza-induced exacerbations of chronic lung inflammation. PLoS One 2014; 9: e98440.

Paget C, Ivanov S, Fontaine J, et al. Interleukin-22 is produced by invariant natural killer T lymphocytes during influenza A virus infection: potential role in protection against lung epithelial damages. J Biol Chem 2012; 287: 8816-8829.

Guillon A, Jouan Y, Brea D, et al. Neutrophil proteases alter the interleukin-22-receptor-dependent lung antimicrobial defence. Eur Respir J 2015; 46: 771-782.

Wiehler S, Proud D. Interleukin-17A modulates human airway epithelial responses to human rhinovirus infection. Am J Physiol Lung Cell Mol Physiol 2007; 293: L505-L515.

Mallia P, Footitt J, Sotero R, et al. Rhinovirus infection induces degradation of antimicrobial peptides and secondary bacterial infection in chronic obstructive pulmonary disease. Am J Respir Crit Care Med 2012; 186: $1117-1124$.

Guinea J, Sandoval-Denis M, Escribano P, et al. Aspergillus citrinoterreus, a new species of section Terrei isolated from samples of patients with nonhematological predisposing conditions. J Clin Microbiol 2015; 53: 611-617.

Delsuc C, Cottereau A, Frealle E, et al. Putative invasive pulmonary aspergillosis in critically ill patients with chronic obstructive pulmonary disease: a matched cohort study. Crit Care 2015; 19: 421.

Jolink $\mathrm{H}$, de Boer R, Hombrink $\mathrm{P}$, et al. Pulmonary immune responses against Aspergillus fumigatus are characterized by high frequencies of IL-17 producing T-cells. J Infect 2017; 74: 81-88.

Werner JL, Gessner MA, Lilly LM, et al. Neutrophils produce interleukin 17A (IL-17A) in a dectin-1- and IL-23-dependent manner during invasive fungal infection. Infect Immun 2011; 79: 3966-3977. with invasive aspergillosis. PLoS One 2015; 10: e0123171.

pentological patients Romani L, Fallarino F, De Luca A, et al. Defective tryptophan catabolism underlies inflammation in mouse chronic granulomatous disease. Nature 2008; 451: 211-215.

Murdock BJ, Falkowski NR, Shreiner AB, et al. Interleukin-17 drives pulmonary eosinophilia following repeated exposure to Aspergillus fumigatus conidia. Infect Immun 2012; 80: 1424-1436.

Barnes PJ. New anti-inflammatory targets for chronic obstructive pulmonary disease. Nat Rev Drug Discov 2013; 12: 543-559.

Sanguinetti CM. N-acetylcysteine in COPD: why, how, and when? Multidiscip Respir Med 2016; $11: 8$. van de Kerkhof PCM, Griffiths CEM, Reich K, et al. Secukinumab long-term safety experience: a pooled analysis of 10 phase II and III clinical studies in patients with moderate to severe plaque psoriasis. J Am Acad Dermatol 2016; 75: 83-98.e4.

03 Vyas P, Vohora D. Innovative targets for asthma and COPD: exploring the existing and screening the new!! Infect Disord Drug Targets 2016; 16: 162-171.

Liljevald M, Rehnberg M, Söderberg M, et al. Retinoid-related orphan receptor $\gamma(\operatorname{ROR} \gamma)$ adult induced knockout mice develop lymphoblastic lymphoma. Autoimmun Rev 2016; 15: 1062-1070. 
105 Maneechotesuwan K, Wongkajornsilp A, Adcock IM, et al. Simvastatin suppresses airway IL-17 and upregulates IL-10 in patients with stable COPD. Chest 2015; 148: 1164-1176.

106 Roy MG, Livraghi-Butrico A, Fletcher AA, et al. Muc5b is required for airway defence. Nature 2014; 505: 412-416.

107 Brusselle GG, Joos GF, Bracke KR. New insights into the immunology of chronic obstructive pulmonary disease. Lancet 2011; 378: 1015-1026.

108 Brightling C, McKenna S, Hargadon B, et al. Sputum eosinophilia and the short term response to inhaled mometasone in chronic obstructive pulmonary disease. Thorax 2005; 60: 193-198.

109 Hurst JR, Vestbo J, Anzueto A, et al. Susceptibility to exacerbation in chronic obstructive pulmonary disease. $N$ Engl J Med 2010; 363: 1128-1138.

110 Pascoe S, Locantore N, Dransfield MT, et al. Blood eosinophil counts, exacerbations, and response to the addition of inhaled fluticasone furoate to vilanterol in patients with chronic obstructive pulmonary disease: a secondary analysis of data from two parallel randomised controlled trials. Lancet Respir Med 2015; 3: 435-442.

111 Bafadhel M, McKenna S, Terry S, et al. Acute exacerbations of chronic obstructive pulmonary disease: identification of biologic clusters and their biomarkers. Am J Respir Crit Care Med 2011; 184: 662-671. 